\title{
Procesos de implementación de herramientas de gestión de la calidad
}

\author{
Implementation process of quality management tools
}

\author{
Carlos Fernando Zuluaga Zuluaga \\ Magíster en Educación y Desarrollo Humano \\ Inventiva Soluciones S.A.S. \\ Manizales, Caldas, Colombia \\ Correo electrónico: gerencia@inventivasoluciones.com
}

Recibido: 18 de septiembre de 2010

Aceptado: 30 de octubre de 2018

\section{Cómo citar este artículo:}

Zuluaga, C.F. (2019). Procesos de implementación de herramientas de gestión de la calidad. Espiral, Revista de Docencia e Investigación, 9(1), 99 - 113.

\section{Resumen}

El objetivo del artículo es describir la investigación centrada en comprender la relación entre herramientas de gestión de la calidad y los resultados en la formación de los estudiantes de los establecimientos educativos de la ciudad de Manizales. Para lograr dicho objetivo, se desarrolló un proceso de carácter cualitativo, con una intencionalidad comprensiva, sustentado en la hermenéutica, mediante la cual se realizó una lectura de un fenómeno de la realidad de los procesos de gestión para interpretar las lógicas de relación entre herramientas de gestión de la calidad y los resultados en la formación de los estudiantes de los colegios y el impacto que ha tenido en la educación.

Los principales hallazgos obtenidos permitieron una aproximación a los principales aportes teóricos enfocados en los procesos de implementación de herramientas de gestión de la calidad y la formación de los estudiantes en establecimientos educativos, sin embargo, encontramos que las investigaciones a nivel nacional son muy pocas y de las cuales muestran una co-relación significativa entre los planteamientos de la normatividad educativa y el discurso. Dando a conocer que, en los últimos años, estos estudios enfatizan los planes de desarrollo de las instancias político-administrativas, pero aun estos planteamientos y discursos no se reflejan de manera efectiva en la transformación social-cultural que requiere el país en relación con los procesos de sistema de gestión de la calidad.

Palabras clave: Gestión de la calidad, hermenéutica, normatividad educativa.
Key words: quality management, hermeneutics, educational regulations.

\section{Introducción}

Esta investigación se pregunta por las relaciones que se establecen entre los procesos de implementación de herramientas de gestión de la calidad, y los resultados de la formación de los estudiantes en establecimientos educativos públicos y privados de la ciudad de Manizales. Este estudio parte del reconocimiento de que la educación es eje de desarrollo de las sociedades del siglo XXI y, como tal, requiere gerenciarse con enfoques de calidad que permita al sistema educativo responder a las necesidades, expectativas y demandas del medio, logrando una satisfacción completa de las comunidades, pero sobre todo, alcanzando una formación integral, de proyección para el nuevo ciudadano que necesita el país. La calidad de la que hablamos es posible mediante una gestión que dinamice, abra puertas, direccione estratégicamente y ponga en escena a cada organización educativa en el contexto social, económico, político y cultural. Mediante una gestión de la calidad la institución educativa podrá ser eficaz, 
eficiente, efectiva y podrá sostenerse, crecer y competir en el mundo de hoy.

Para alcanzar esta calidad de la que hablamos ha emergido una marcada tendencia en los establecimientos públicos y privados de educación inicial, básica y media para implementar diferentes modelos y sistemas de gestión de la calidad que en algunos casos son certificados o acreditados por organismos reguladores nacionales e internacionales. En Colombia, los entes acreditadores reconocidos por el Ministerio de Educación Nacional -MEN son norma ISO 9001, reconocida mediante Resolución del MEN n' 4434 del 8 de agosto de 2006, que establece que en el país el organismo nacional de acreditación de Colombia - ONAC es el responsable de autorizar y vigilar a las entidades certificadoras, y que el proceso de certificación en educación es orientado por la guía técnica colombiana GTC-200', entre otras, las cuales se abordaran de manera específica en los antecedentes y marco teórico.

Siguiendo con el planteamiento de la investigación realizada, en la práctica educativa existe preocupación y una mirada crítica desde las dinámicas de la educación, la pedagogía y la gestión y su impacto en la formación de los niños y niñas que requiere el país y el mundo. Los procesos de gestión de la calidad que se diseñan implementan y mantienen en los establecimientos educativos están impactando, fortaleciendo el sistema educativo desde lo administrativo y directivo. A su vez, se identifican factores por potenciar en la gestión del aula, desde los agentes educativos, directivos y otros actores sociales que aportan al sistema educativo a legitimarse.

Por lo anterior, esta investigación a punto a reflexionar los sistemas de gestión de calidad, también identifica puntos articuladores entre los lineamientos y políticas y las prácticas de

1 Guía Técnica para la Implementación de la Norma ISO 9001 en establecimiento de educación formal en los niveles de preescolar, básica, media y en establecimientos de educación no formal (ICONTEC, 2005). gestión que realizan las instituciones educativas. Entre otros puntos, los que aporten a la resignificación de la educación, partiendo de la reflexión, para llegar a nuevos sentidos de las educaciones, apuntando a la gestión de la calidad.

Existen instituciones educativas que potencian una gestión de calidad integral y que trascienden los enfoques técnicos e instrumentales de la calidad, es necesario rescatarlas, potenciarlas y proyectarlas a partir del reconocimiento de sus buenas o malas prácticas y experiencias significativas que permita posteriormente replicarse e integrarse a alguno de los modelos existentes.

Por lo anterior enunciado, esta investigación se aventuró a reconocer las relaciones que se dan entre los procesos de gestión de la calidad y los resultados de los procesos de formación de los estudiantes para superar esa brecha consistente en una concepción de que construir sentidos logra una gestión de calidad integral que, a partir de un enfoque sistémico o complejo de la organización educativa, permee todas las áreas, procesos y actores de la comunidad educativa.

La revisión bibliográfica, teórica, conceptual $y$, sobre todo, los antecedentes de investigaciones similares o procesos de intervención institucional para implementación de sistemas de calidad en instituciones educativas, identificaron algunos planteamientos y tendencias que sustentan la necesidad de iniciar procesos investigativos específicos en el área, que permitan avanzar en el conocimiento, desde la comprensión de las realidades de los sistemas de gestión de la calidad y su relación con los resultados de la evaluación de la formación de los estudiantes, dentro de los hallazgos encontrados, los más representativos son los siguientes:

- Es necesario articular el sistema de gestión de la calidad al Proyecto Educativo Institucional, de manera que sus herramientas faciliten su puesta en marcha. Otro ha- 
llazgo es la no continuidad en el proceso de acompañamiento por parte de las Secretarías de Educación, y la gestión de recursos para la certificación lleva a que los establecimientos educativos abandonen los procesos y herramientas de calidad y se pierda el camino recorrido.

- La certificación de calidad no necesariamente es garante de la calidad de la educación, es necesario consolidar procesos de transformación cultural que redunden en beneficio de la formación. Así mismo, la rotación del personal, especialmente de los directivos y docentes, incide directamente en el mantenimiento del sistema de gestión de la calidad y en la posibilidad de mantener su certificación.

- En las investigaciones a nivel internacional y locales, en sus hallazgos coinciden en que se deben contextualizar las herramientas gerenciales a las dinámicas institucionales, de manera que hagan parte de la cotidianidad y de la gestión de su proyecto educativo institucional. Se deben seguir los procesos de medición, análisis y mejora continua que requieren del acompañamiento permanente en los establecimientos educativos. Como también es necesario trascender la medición de la calidad de la formación de los establecimientos educativos basada en los resultados de las pruebas externas, para avanzar en criterios como la pertinencia. Como aspecto significante, se resalta el desempeño de los egresados en el sector productivo o la universidad, el alcance del perfil de estudiante y el desarrollo de la capacidad crítica-reflexiva, los procesos de participación, como criterios que deben atenderse e instalarse en el currículo y en los planes de mejora de cada institución.

Como antecedente significativo, en Manizales y los municipios del departamento de Caldas, la Universidad Católica de Manizales (UCM) ha venido ejecutando proyectos de investigación, desarrollo y procesos de formación continua en gestión de la calidad con establecimientos educativos públicos y privados de la ciudad. Esta experiencia se constituye en antecedentes del proyecto de investigación, por la necesidad de dar un paso más en la comprensión de las lógicas de diseño, implementación, mantenimiento y mejora continua de los sistemas de gestión de la calidad como posibilidad de puesta en marcha del Proyecto Educativo Institucional. Entre ellas tenemos: La investigación Sentidos de la gestión de la calidad en los directivos docentes de las instituciones educativas oficiales urbanas de Manizales, por los autores Zuluaga, Rivera y Quintero (2011). El objetivo que se trazó esta investigación se orientó en comprender el significado que tiene para los directivos, docentes, la gestión de la calidad de las instituciones educativas oficiales, urbana de Manizales. La metodología estuvo enmarcada en el enfoque cualitativo, desde la teoría fundada en combinación con técnicas como es la escala de Likert, el grupo focal y la revisión documental. Los resultados más representativos fueron aceptación y valoración en torno a la gestión de la calidad posterior a un proceso crítico a directivos y comunidades educativas relacionadas con la gestión. Otros hallazgos hacen referencia a que la concepción de calidad se ha transformado, impactando con ello la cultura organizacional, la incorporación del ciclo de la mejora continua, el fortalecimiento del liderazgo y trabajo en equipo.

Otra investigación destacada, como antecedente en el presente estudio, fue la investigación de tipo documental, denominada: Investigación documental sobre calidad de la educación en instituciones educativas, realizada en el 2015 por Durley Bernal Suárez, Mary Luz Martínez Pineda, Angélica Yulieth Parra Pineda, docentes, investigadoras de la Universidad Santo Tomás; esta investigación tuvo como objetivo principal, establecer el estado de la investigación sobre calidad educativa a partir de los artículos publicados en las bases de datos (Dialnet, Doaj, 
E-revistas, Latindex, Rebiun, Recolecta, Redalyc y Scielo) en los últimos 10 años. Este estudio se desarrolló con un enfoque cualitativo. Algunos de los hallazgos fueron los siguientes: la calidad educativa es un concepto macro, tomado desde las entidades gubernamentales, en el cual se hace importante evidenciar calidad en los entornos educativos en relación con los requerimientos y políticas nacionales e internacionales. Así mismo, el estudio resaltó que la calidad de la educación conlleva vislumbrar los propósitos de las instituciones educativas en un nivel meso, teniendo en cuenta la calidad de los procesos de enseñanza-aprendizaje que reflejan ante los entes reguladores de control y evaluación; y como un último concepto, a nivel micro, sobresale "educación de calidad", como aquella que se desarrolla en el aula a partir del currículo y las planeaciones que orienta el educador con el único objetivo de fortalecer en el estudiante sus habilidades y competencias, siendo así, el educador, el responsable directo de la calidad resultante.

Por último, una investigación internacional: Estilos de liderazgo y resultados del sistema de medición de la calidad de la educación: un estudio empírico en los colegios básicos de la ciudad de Arica, Chile, desarrollada por Liliana PedrajaRejas, Emilio Rodríguez-Ponce, Manuel Barreda Olavarría, Omar Sagredo Núñez y Cristian Segovia León (2007). Los resultados sugieren que el liderazgo en los colegios considere que los agentes institucionales y líderes deben compartir la misión y la visión con su equipo técnico y sus profesores, logrando que los equipos técnicos y docentes apliquen con motivación los objetivos planteados.

Los anteriores estudios fueron muy significantes para la investigación, identificando que el liderazgo de los agentes educativos, como son los directivos de las instituciones, son muy importantes para el sistema de gestión de calidad, en compañía de un equipo de trabajo participativo y comprometido, que permita el mejoramiento de la calidad de la enseñanza en el aula. También, estas investigaciones sugieren análisis continuos en relación con los factores endógenos y exógenos que se tejen en una institución, dando a conocer en el contexto las herramientas gerenciales implementadas y la manera en que se facilite o limite la implementación y la relación con los factores internos y externos.

\section{Metodología}

El estudio se realizó desde un enfoque cualitativo, con una intencionalidad comprensiva, con el fin de analizar e interpretar las lógicas de relación entre herramientas de gestión de la calidad y los resultados en la formación de los estudiantes de los colegios, desde una revisión e interpretación del contexto legal, normativo, teórico, conceptual, estratégico de la implementación de dichas herramientas en aproximadamente tres establecimientos educativos de la ciudad de Manizales del sector público y privado. Esta intencionalidad del proyecto de investigación está enmarcada en la visión hermeneútica del contexto educativo, caraterizada por ser una corriente filosófica, que busca interpretar y comprender, permitiendo con ello, develar, las realidades subjetivas, psicológicas del ser humano.

La pretensión de lo anterior, en el marco de la investigación, fue identificar y relacionar las tendencias en el contexto normativo, los modelos teóricos y conceptuales que sustentan las diferentes herramientas gerenciales de la calidad, su apropiación e implementación en el contexto educativo desde las percepciones, saberes, prácticas y experiencias de los diferentes actores involucrados en este proceso. En este sentido, se relacionó el funcionamiento de las herramientas de sistemas de gestión implementadas en las instituciones educativas, desde el actuar cotidiano de los diferentes actores implicados, partiendo de la comprensión de las realidades y problemáticas, en el marco de las diversas apuestas teóricas, normativas que se tienen y relacionándola con las prácticas de gestión. 


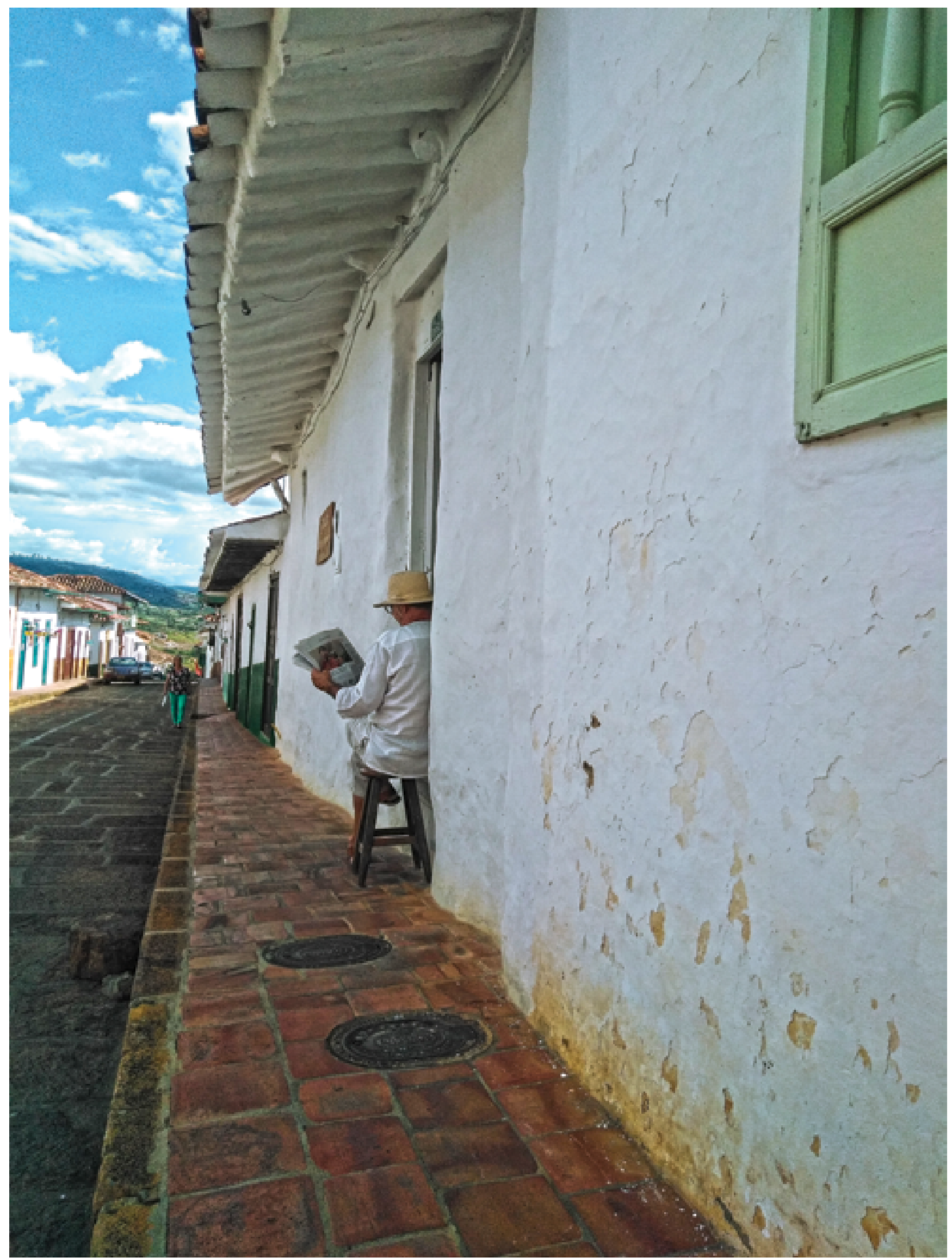


El diseño que se utilizó fue la hermenéutica, la cual realiza procedimientos sistemático cualitativo para generar una teoría que explique en un nivel conceptual una acción, una interacción o un área específica, cuyo propósito es desarrollar teoría basada en datos empíricos" (Hernández y otros, 2010, p. 492). En este estudio, se implementó el diseño sistemático, con el fin de construir las categorías de análisis a partir de los datos recolectados por la investigación.

\section{Fases de la investigación}

\section{Descriptiva}

Momento en el que se hizo la delimitación del campo de estudio, y con ello, el acercamiento de las realidades educativas, así mismo, se realizó la triangulación de métodos de recolección de los datos, por medio de la entrevista a profundidad a los líderes del sistema de gestión de la calidad, en este caso fueron los directivos, coordinadores académicos y docentes de área. El tipo de entrevista fue con preguntas abiertas y semiestructuradas. Estas entrevistas se grabaron en audio, para su posterior digitación, clasificación y análisis de información. En esta fase se revisaron las fuentes primarias, como documentos, registros o materiales elaborados por los directivos, docentes y coordinadores, en relación al Proyecto Educativo Institucional, medición y análisis de indicadores, evidencias de la evaluación institucional y de las acciones de mejora, planes de mejoramiento institucional, gestión del talento humano, gestión de espacios y recursos, estructura académica de formación relacionada con el objeto de estudio de la investigación, con el fin de identificar los antecedentes del ambiente, las experiencias relacionadas, vivencias o situaciones y su funcionamiento. Por último, se hicieron sesiones en profundidad con grupos focales, con el fin de validar las categorías emergentes, aclarar información relevante y lograr mejor comprensión de la realidad de los sistemas de gestión de la calidad en el contexto educativo.

\section{Interpretativa}

Se realizó el análisis de los datos en el marco del diseño sistemático, los cuales fueron: Codificación abierta, con la que se revisan todos los segmentos del material para analizar, generando categorías iniciales. Codificación axial, con la que se agrupan los datos identificados, separados y codificados por el investigador, para crear vínculos entre categorías y temas, y construir un modelo del fenómeno estudiado (esquema). Codificación selectiva, en la que el investigador regresa a las unidades o segmentos y los compara con su esquema emergente para fundamentarlo, de lo cual derivará una narración que vincule las categorías y describa el proceso o fenómeno estudiado, utilizando como herramienta el análisis cualitativo de los mapas mentales. En este momento se consolidaron las categorías, desarrollando el ejercicio de integrar los segmentos que comparten la misma naturaleza, significado y características y así identificar las relaciones que se establecen entre ellas, con el fin de facilitar el análisis e interpretación de los datos.

\section{Construcción de sentido de las categorías}

Se identifican las relaciones existentes entre ellas, con el fin de develar los hilos conductores que relacionan las diferentes categorías construidas en la fase anterior, permitiendo la comprensión de las narrativas de los entrevistados en relación con la gestión de la calidad y los hilos que se tejen en la relación con los resultados en la formación de los estudiantes de los establecimientos educativos de Manizales.

Este estudio abordó tres establecimientos educativos públicos de la ciudad de Manizales, los cuales implementan el sistema de gestión de la calidad en sus organizaciones, con certificación de calidad en instancias nacionales o internacionales. En este sentido, se realizó la búsqueda en bases de datos para identificar las instituciones que cumplen este requisito. Los cuales fueron: 
- Un colegio privado con ISO $9001=$ Colegio Seminario Redentorista San Clemente María Hofbauer.

- Un colegio privado con $\mathrm{EFQM}=$ Colegio Santa Inés (que cuenta además con el Modelo ISO 9001).

- Un colegio público con Modelo De y Alegría = IE Fe y Alegría, la Paz (Manizales).

De cada una de estas instituciones participaron los directores, coordinadores del área de calidad y los docentes, como agentes educativos claves para develar la relación entre gestión de la calidad y resultado de evaluación de los estudiantes. Este estudio se desarrolló teniendo presente el periodo del 2014 y 2017.

\section{La Unidad de análisis, se tuvieron presentes los siguientes criterios}

\section{Modelo de gestión de la calidad}

- Selección del modelo de gestión (ISO 9001, EFQM, Fe y Alegría).

- Criterios para la elección del modelo de gestión y la relación con los resultados de la formación.

- Estrategias utilizadas para la formación, diseño, implementación y certificación del modelo de gestión.

- Percepción general acerca del Modelo de Gestión de la Calidad implementado y certificado y su relación con los resultados académicos institucionales.

\section{Planeación institucional}

- Tipo de planeación seleccionada.

- Estrategias y formas de diseño e implementación del tipo de planeación.

- Estrategias para el liderazgo y el trabajo en equipo.
- Relación entre la planeación, los fines de la educación y el horizonte institucional.

- Estrategias de difusión y apropiación del horizonte institucional.

- Compromiso de los actores con el horizonte institucional.

- Planificación del mejoramiento de los resultados académicos institucionales: estrategias, actividades, herramientas.

\section{Evaluación institucional}

- Evidencias del desarrollo y mejoramiento a través de los años de implementación del modelo de gestión.

- Resultados históricos de la autoevaluación institucional.

- Indicadores y metas institucionales.

- Estrategias de medición y análisis de metas e indicadores.

- Aporte de las metas e indicadores al mejoramiento institucional.

- Tipos de metas e indicadores que potencian los resultados en la formación

- Estrategias de evaluación del modelo de gestión.

- Relación entre las estrategias de evaluación del modelo de gestión y las acciones de mejoramiento.

- Estrategias para el seguimiento a la planificación.

- Medición de la percepción de la comunidad educativa.

- Análisis de los resultados de las pruebas académicas internas y externas.

- Relación entre la evaluación y el mejoramiento académico de los estudiantes. 


\section{Mejora continua}

- Relación entre la evaluación y la mejora continua.

- Enfoque hacia la eliminación de las causas de las oportunidades de mejora identificadas.

- Acciones de mejora planificadas en el tiempo de implementación y mantenimiento del modelo de gestión.

- Relación entre el modelo de gestión y el mejoramiento institucional.

- Acciones de mejora de los resultados académicos de los estudiantes.

- Relación entre la mejora continua y los resultados académicos de los estudiantes.

\section{Diseño curricular}

- Lógicas y racionalidades para el diseño curricular.

- Estrategias para la articulación, transversalización y flexibilización curricular.

- Estrategias para la incorporación de los resultados de las pruebas externas e internas en el diseño curricular.

- Diseño de currículo por competencias.

- Herramientas del modelo de gestión que incide en el diseño curricular.

- Relación entre las herramientas y los resultados del diseño curricular.

- Relación entre el diseño curricular y los resultados académicos de los estudiantes.

\section{Desarrollo curricular}

- Estrategias para el seguimiento a la eficacia del diseño curricular.

- Uso de mediaciones pedagógicas.

- Modelo pedagógico.
En este proyecto emergieron nuevas categorías, los cuales se clasificaron según los tipos propuestos por Creswell, como posibilidad de organización de la información.

Por último, el plan de análisis se realizó por medio de una matriz de información, con el fin de organizar las categorías, identificando las tendencias. Para la matriz se tuvo presente los siguientes criterios: evidencia empírica, categóricas, tendencia, observación y análisis.

\section{Hallazgos}

En la investigación acogimos esta frase como horizonte, al cual quiso llegar el presente estudio: "La cultura organizacional tiende a ser considerada como un recurso valorable que puede convertirse en una fuente especial de ventaja competitiva... Un componente significativo de ella es la cultura de la calidad" (Anónimo, 2011). Este producto resulta de jornadas de lectura y de un trabajo comprometido con las instituciones educativas y los participantes.

Las categorías que se elaboraron mediante triangulación teórica, correspondiente a la revisión de autores, antecedentes y al establecimiento de categorías de análisis que permitieron definir dichas tendencias o categorías (Álvarez y Topete, 2004; Pérez, 2015). La información derivada de la primera y segunda fase del estudio fue analiza mediante las categorías conceptuales con relación al sistema de gestión de la calidad y a las herramientas de gestión, como también la unidad de análisis, en este caso, las instituciones educativas.

Para aproximarnos al componente teórico y metodológico tuvimos planteamientos de autores como Kent y De Vrie (2000), quienes identifican cinco concepciones distintas de calidad, como también las implicaciones y las relaciones que se establecen en la calidad, como son las políticas de la educación. Así mismo, la gestión de la calidad, desde Fontalvo (2002). Los principios de gestión de la calidad propuestos 
por la Norma ISO para sustentar la cultura de la calidad, retomados en el documento de gestión por procesos de Pérez Hernández (2010). Partiendo de los principios que sustentan las políticas y la gestión de la calidad en las organizaciones, desde Quiñonez (2010), quien clasifica los modelos de calidad de las instituciones educativas en dos enfoques para administrar e implementar la cultura de la calidad: Hoshing Planning (modelo japonés de administración por políticas); Ciclo PDCA (planificar-hacercomprobar-actuar) o ciclo PDSA (planificar-hacer-estudiar-actuar) o ciclo PHVA (planear, hacer, verificar y actuar) ciclo de shewart/Deming, Diagrama de Dispersión. Evaluación de calidad, entre otras herramientas que son significativas para la comprensión y análisis del sistema de gestión.

Respecto a los modelos de certificación de la calidad en Colombia, el Ministerio de Educación Nacional reconoce los modelos de gestión que aplican los establecimientos educativos privados de preescolar, de acuerdo con lo establecido en el Decreto 529 de 2006 y la Resolución 4434 del mismo año. Para el reconocimiento de un modelo se requiere que: 1 . se aplique internacionalmente (al menos en cinco países); 2. integre los conceptos de administración y aseguramiento de calidad; 3 . tenga mecanismos para asegurar la independencia de la evaluación; 4. suponga una autoevaluación con exigencias al menos iguales a las requeridas en la Guía 4 MEN para clasificarse en el régimen de Libertad Regulada y, 5. se aplique de manera específica en el sector educativo. En este sentido, El Ministerio de Educación Nacional, con la Resolución No. 4434 del 8 de agosto de 2006, estableció las condiciones para la validación de los modelos de reconocimiento de gestión de calidad como requisito para aplicar el régimen de libertad regulada de tarifas, y precisa el alcance de la certificación del sistema de gestión de calidad, por medio del cual plantea la necesidad de verificar que los entes acreditadores de calidad de las instituciones educativas cumplan con algunos requisitos mínimos en los cuales incluyan los conceptos básicos del aseguramiento de la calidad (Resolución No. 4434 de 8 de agosto de 2006).

Partiendo de lo anterior, el Ministerio de Educación Nacional reconoce a las entidades certificadoras para que abalen algún modelo internacional de gestión de la calidad en las instituciones de educación del país, como ISO 9001:2008, EFQM, NEASC-CIS, AdvancED, Modelo Fe y Alegría y Modelo PCI A.

Estas apuestas teóricas y los hallazgos identificados en el presente estudio permiten triangular el conocimiento para la implementación de sistemas de calidad en instituciones educativas. En este marco de ideas, en el presente trabajo, se identificaron categorías y subcategorías, las cuales sustentan la necesidad de iniciar procesos investigativos específicos en el área, que permiten transcender el conocimiento, desde la comprensión de las realidades y sentidos de los sistemas de gestión de la calidad y las relaciones que se establecen, resultados de la evaluación de la formación de los estudiantes, dentro de los hallazgos se identifican seis categorías grandes en el sistema de gestión de calidad.

\section{Mejora continua}

Se reconoce la necesidad de implementar un proceso de mejoramiento continuo, para alinear los procesos de formación hacia el cumplimiento de las metas propuestas. Además, la necesidad de realizar planes de mejoramiento en cada área de gestión, dependiendo de las debilidades encontradas de la institución. Los planes de mejora continua hacen parte de los principios de gestión de la calidad propuestos por la ISO para sustentar la cultura de la calidad, retomados del documento de gestión por procesos de Pérez (2010, pp. 229-232). Para el autor, este término, hace parte de reconocer las necesidades de hacer mejoras constantemente, teniendo en cuenta los detalles de cada 
proceso, la intención es hacer mejoras o arreglos como requisito fundamental para lograr el éxito a largo plazo, en este sentido la mejora continua se convierte en un propósito para la organización.

\section{La evaluación}

Se considera un insumo fundamental para diseñar los planes de mejora y la planeación institucional. Así mismo, el Sistema de Gestión de la Calidad aporta diversas herramientas, tales como: herramientas para evaluación, chequeo de actividades por proceso para hacerles seguimiento, chequeo de los indicadores por resultado, guía de evaluación del plan de mejora, herramientas de registro de percepciones de acuerdo con el momento en que uno esté. La cultura de la evaluación constante permite la apropiación de herramientas de gestión y la apertura a la autoevaluación y a la evaluación, para poder identificar aspectos por mejorar desde las diversas perspectivas. En este sentido, se valora la autoevaluación como motor para cualificar el desempeño permanente por parte de los docentes.

\section{Evaluación integral permanente al diseño curricular}

Esta tendencia alienada a procesos de auditoría interna como un proceso importante para evaluación y seguimiento a los procesos institucionales.

\section{Participación de la familia y la comunidad}

La participación de la familia y la comunidad en los procesos de evaluación, así como en toda la dinámica institucional. Esta última, la comunidad, permite tener una presencia institucional, como institución educativa diferente a lo que tienen muchas otras, porque ellas tienen más labor social, entonces la imagen que tiene la institución afuera es de credibilidad, en el sentido humano, en lo social.

\section{Planificación}

Se reconoce la gestión de riesgo como una estrategia importante de planificación, Como estrategia de diseño e implementación de los planes de mejora se genera una línea estratégica, el plan de mejora está enfocado a los procesos de convivencia, de interacción entre ellos, con ellos y con el entorno y a los procesos de resultados académicos con pruebas externas. Otra línea estratégica son los resultados académicos, como también otros actores sociales, como los padres de familia, la comunidad educativa, los docentes; los cuales se convierten en grupos de reflexión que se organizan con todos los componentes de la comunidad educativa. Se reconoce que las líneas estratégicas deben ser diseñadas en forma participativa.

\section{Equipos de mejora}

El trabajo participativo y colaborativo refuerza la apropiación de los procesos de gestión de la calidad. Los equipos de mejora se convierten en una estrategia para articular el trabajo en la institución, resaltando el liderazgo del Comité de Calidad, Consejo Académico, como instancia fundamental para retroalimentar y apoyar la gestión curricular desde el diseño, ayudando en la evaluación, análisis de los procesos por desarrollar.

Para fomentar la participación de los diferentes estamentos de la institución en los procesos de mejoramiento continuo se establecen como estrategia de trabajo los grupos de reflexión, estos como escenarios que permiten conocer las prácticas educativas de los estudiantes y el contexto familiar y otros actores sociales. La estrategia de equipos de reflexión está contemplada en el Sistema de Gestión de la Calidad. Otro proceso de participación que reconocen en el marco de los equipos de mejora es el Consejo Académico, como instancia fundamental para retroalimentar y apoyar la gestión curricular desde el diseño. 


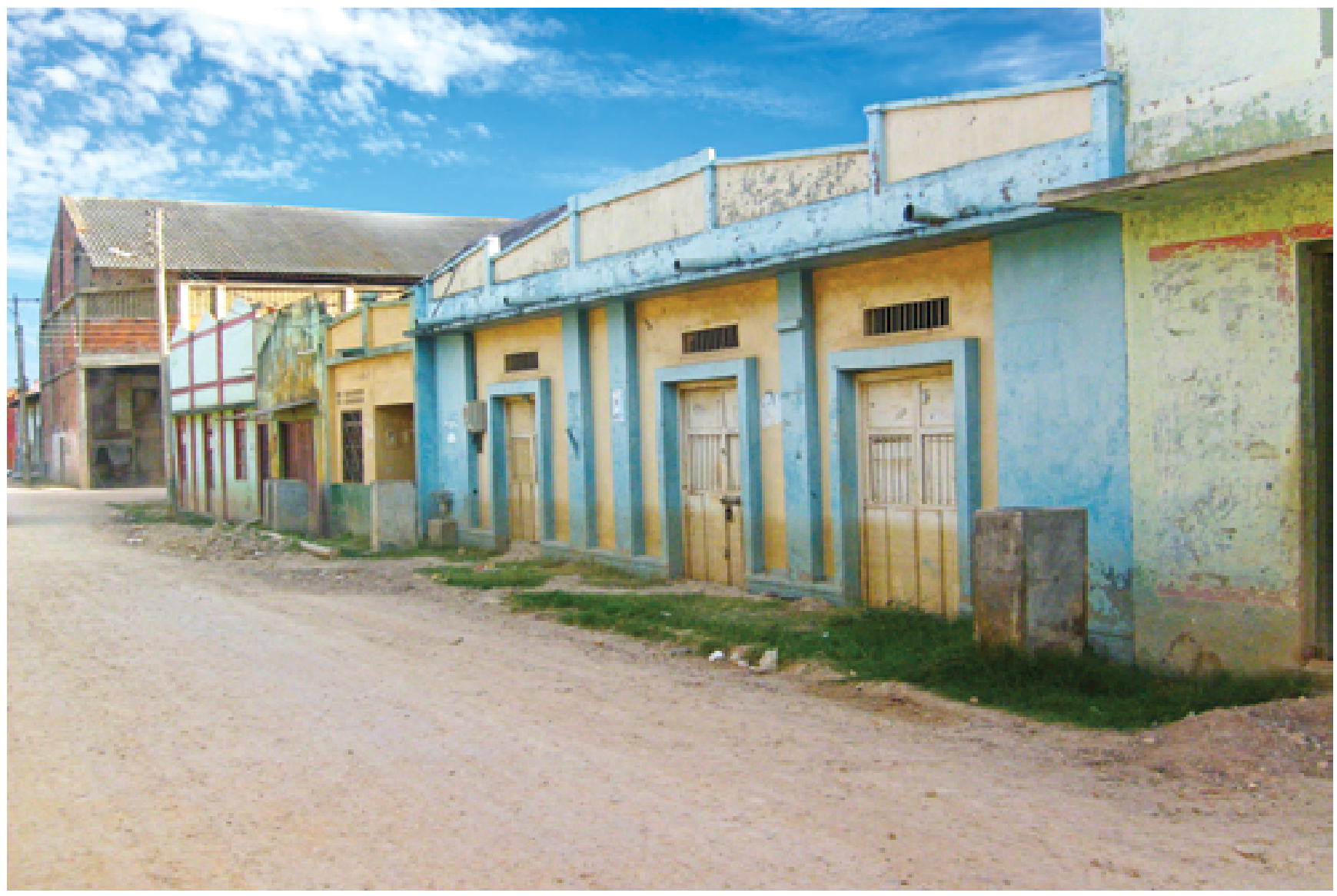

Otro factor importante de los resultados y de los cuales se valora institucionalmente, es el trabajo en grupos de reflexión, estos, como escenarios participativos, que permiten el conocimiento de los estudiantes y el contexto familia. A su vez, se identifica que esta estrategia se contempla en el sistema de gestión de calidad.

\section{Gestión curricular}

En las instituciones se reconoce la necesidad de realizar la planeación y gestión curricular, partiendo de los diagnósticos, las tendencias teóricas, normativas y contextuales, además de las necesidades y expectativas.

Otro requisito fundamental para el diseño curricular es la coherencia entre el modelo pedagógico, las estrategias formativas y la mediación pedagógica, respondiendo a las necesidades del contexto y Modelo Pedagógico y lineamientos institucionales. La gestión curricular se reali- za partiendo de los lineamientos, ejecutándose con el direccionamiento y gestión de los líderes de cada área. Estos procesos curriculares se desarrollan desde el plan de mejora, y su validación se hace de manera conjunta.

Otras narrativas apuntan a que la gestión curricular la reconocen como factor facilitador de los procesos en desarrollo de la institución educativa, así mismo, permite medir eficacia del currículo para poder verificar la coherencia entre lo planificado, lo ejecutado y los resultados reales. Como también mide la trazabilidad de los procesos y el acompañamiento y seguimiento de los directivos para verificar el cumplimiento de las metas propuestas. También permite fortalecer los procesos académicos enfocados a los resultados y al impacto deseado, no solo en el proceso formativo del estudiante, sino también en el contexto. La eficacia en la gestión curricular también se mide desde la trazabilidad 
de los procesos y el acompañamiento y seguimiento de los directivos para verificar el cumplimiento de las metas propuestas.

\section{Integración del Sistema de Gestión de la Calidad}

Esta categoría como posibilidad de cualificación de los procesos pedagógicos y académicos, que también influye como un factor fundamental en los procesos es la articulación curricular para desarrollar temáticas articuladas en las materias que se puedan integrar, permite mayor optimización de los procesos y el logro de aprendizaje significativo. Es importante esta articulación curricular por parte de los estudiantes y sus padres de familia.

Otro aspecto significativo en esta categoría es la de integrar a la familia para fomentar la inclusión escolar. La inclusión se percibe como una cultura institucional que implica trascender la valoración del ser humano exclusivamente desde la productividad académica, para asumirlo como un ser humano integral que merece respeto, no solo por parte de docentes, sino también de los otros compañeros del aula.

\section{La flexibilidad curricular}

La flexibilidad hace vida en los procesos formativos cuando se les hacen adecuaciones y platean estrategias, mediaciones a nivel de los logros, de acuerdo con las posibilidades y expectativas de los jóvenes, lo cual implica una sensibilización y formación de los docentes. Como aspecto importante en esta categoría, está el diagnóstico o caracterización integral de los niños en condiciones especiales, es fundamental para poder tener flexibilidad curricular acorde con las necesidades y condiciones reales. Esta caracterización se hace por medio del SIMAT.

\section{El bilingüismo}

El bilingüismo aparece como una tendencia en los colegios analizados desde la integralidad del currículo, se propone formar a seres humanos excelentes, lo cual debe estar en coherencia con el trabajo cotidiano, según lo exponen los actores sociales. El trabajo colaborativo y en equipo surge como otra estrategia para el diseño curricular integral.

\section{Gestión del PEI}

El diseño del PEl parte de la revisión de la pertinencia y coherencia del horizonte institucional. Como estrategia de funcionamiento de los procesos institucionales está la articulación del PEI con los lineamientos de calidad.

Otro aspecto relevante de esta categoría es el reconocimiento que le hacen los docentes y directivos al trabajo, con la lógica del mejoramiento continuo, y el fomento de la calidad permite mayor coherencia, integración y efectividad en el logro de los resultados. Para alcanzar la implementación de los procesos de calidad, los actores sociales reconocen los siguientes insumos: compromiso de todos y un trabajo en equipo de manera colaborativa. Como segundo, se debe de hacer un proceso de conocimiento, cualificación constante. Como también, el docente se debe involucrar en cada uno de los procesos de planificación, planes de mejora. El seguimiento constante de los procesos. Se debe contar con un asesor permanente de calidad. Realizar jornadas pedagógicas. Involucrar a la familia y la comunidad.

\section{Ambiente escolar}

El ambiente escolar es reconocido por los actores sociales, casi que exclusivamente desde la categoría de convivencia. Un criterio de calidad importante no solo es la valoración externa sino la disminución de los casos de dificultades de convivencia en el aula. El ambiente escolar sano se debe dar como la posibilidad de potenciar el aprendizaje y lograr mejores resultados académicos en el desempeño escolar. En síntesis, se reconoce el ambiente escolar como escenario fundamental del aprendizaje que se 
debe evaluar, analizar y potencializar, como prerrequisito fundamental para lograr un buen desempeño escolar.

\section{Gestión del talento humano}

La capacitación permanente del talento humano aparece como una categoría fundamental para lograr planeación curricular adecuada y pertinente. Esta categoría se resalta como un requisito para alcanzar buenos resultados en el Sistema de Gestión de la Calidad, el cual debe ser liderado estratégicamente desde la Dirección de la institución.

Para el fomento de los procesos de calidad aparece como un factor importante la cualificación del personal de la institución. En el marco de esta categoría aparece la práctica de selección del talento como proceso de gestión de la calidad, definir perfiles, como también facilita la gestión curricular. Otros componentes son la evaluación y el seguimiento continuo del trabajo de los docentes, se reconocen como un factor fundamental para garantizar la calidad en el proceso formativo. Como también, la gestión de la calidad, la cual debe ser liderada estratégicamente desde la Dirección de la institución. Por último, la estrategia del apoyo de unos docentes a otros en el proceso de inducción en la institución como estrategia de gestión del talento humano.

\section{Conclusiones}

Los sistemas de gestión de la calidad y la relación con los resultados de evaluación, se convierte en una necesidad para las instituciones educativas, a su vez, en grandes desafíos para los agentes institucionales, decisores de política pública y toda comunidad educativa que participa en este proceso. Permitiendo, con ello, incidir en la formulación de nuevos lineamientos para el Ministerio de Educación. La investigación como camino reflexivo que invita de manera continua resignificar los sentidos hacia los cuales se orientan y se pone en práctica la educación en Colombia. En ese sentido, las problemáticas y realidades que se tejen y se establecen desde lo educativo influyen en lo social y cultural de un país, afectando o fortaleciendo la condición humana.

Por lo anterior, la investigación basada en las categorías y tendencias identificadas que permitieron hacer lecturas de las realidades educativas en el marco de la gestión de la calidad y de su relación, concluye:

- La gestión directiva, administrativa, académica y gestión comunitaria va de la mano con la gestión de la calidad en el marco de los lineamientos establecido por el Ministerio de Educación. Por ello se hace necesario potenciar el trabajo en equipo y cooperativo, fomentando el liderazgo, la autotelia, capacidad de agencia de cada uno de los que participa en la construcción de conocimiento.

- Teniendo presente la anterior conclusión, se hace necesario crear y potenciar desde la práctica educativa, generar espacios de diálogos constructivos, los cuales inviten a realizar lecturas de las realidades que se establecen en el establecimiento educativo, las relaciones que se tejen con los estudiantes, el conocimiento que se tiene de ello, y sus entornos familiares, las prácticas pedagógicas, el conocimiento de los lineamientos institucionales. Son temas de interés de toda una comunidad educativa para poner en conversación desde los grupos reflexivos.

- Las auditorías, tanto internas como externas que se realizan en las instituciones educativas, se convierten en una necesidad y desafío constante para la comunidad educativa. Puesto que la auditoría permite reflexionar los procesos que se desarrollan, retroalimentando, haciendo diagnósticos situacionales o la actualización de estos, así como la realización de estrategias de mejora que ayudan en los 
procesos de la gestión de la calidad.

- La planificación institucional también es importante resaltar, ya que permite generar horizontes organizados que optimizan los procesos desarrollados y por ejecutar. Esta planificación se debe poner en acción, obedeciendo a las exigencias realizadas por el Sistema de Gestión de Calidad.

- La medición de la calidad de la educación es una categoría que se debe de resaltar, puesto que este proceso permite identificar los procesos desarrollados por la institución, dar organización a los mismos, fomentar la planificación con base en los procesos de mejora, entre otros. Lo anterior contribuye a la identificación de los resultados y al impacto generado.

- Se hace imperativo que se articulen los procesos realizados en relación con el sistema de calidad con los proyectos institucionales, desde una coherencia teórica y práctica. Con el fin de identificar factores potencializadores y factores por potencializar de los proyectos, con los sistemas de calidad y de esta manera realizar planes de mejoramiento para su funcionamiento.

\section{Referencias}

AdvancED. Nuevos componentes de acreditación. Puerto Rico. 2012.

Aldana de V., L. Á. (s.f.). Administración por calidad. Universidad de la Sabana. Recuperado de http:/site.ebrary.com/ id/10565646?ppg=26 y ppg $=40$.

Álvarez, I., Topete, C. (2004). Búsqueda de la calidad en la educación básica. Conceptos básicos, criterios de evaluación y estrategia de gestión. Revista Latinoamericana de Estudios Educativos, XXXIV(3), 7. Recuperado de http://www.redalyc.org/articulo.oa?id=27034302

Barrera, F., Maldonado, D., y Rodríguez, C. (2012, octubre). Calidad de la educación básica y media en Colombia: Diagnóstico y propuestas. Serie Documentos de Trabajo, No. 126 , pp. 6-7.

Brooking, A. (1997). El capital intelectual. Barcelona: Ediciones Paidón Ibérica.

Cajiao, F. (2008). Evaluar es Valorar. Plan decenal en acción evaluación del aprendizaje y calidad de la educación. Lineamientos de discusión (pp. 16 y 29).
Cazorla, J. (2004). Sistema de gestión de la calidad. España: Emagister.

Deming, W. (1989). Calidad, productividad y competitividad. La salida de la crisis. Ediciones Díaz de Santos.

Fontalvo, T. J. (2000). La gestión avanzada de la calidad: metodologías eficaces para el diseño, implementación y mejoramiento de un sistema de gestión de la calidad. Corporación para la Gestión del Conocimiento ASD. Recuperado de https://scholar.google.com/citations?user=DBaJO6c AAAAJ\&hl=es

García, S., y Maldonado, D. (2014, marzo). Propuestas para el mejoramiento de la educación preescolar, básica y media en Colombia. Cuadernos de Fedesarrollo, Nro. 49, 22-23. Edición Convocatoria Germán Botero de los Ríos. Debates Presidenciales.

Hernández, R., y otros. (2008). Metodología de la investigación (4a ed., pp. 668-692). Ed. Mc Graw-Hill.

ICONTEC Internacional. (2011). Guía Técnica GTC colombiana 200. Guía para la implementación de la norma ISO 9001 en establecimientos de educación inicial y formal en los niveles de preescolar, básica y media. (1 a actualización, p. 5).

ICONTEC. (2009). Normas fundamentales sobre gestión de la calidad y documentos de orientación para su aplicación ( $2^{\text {a }}$ ed., pp. 9 y 11 ).

Instituto Colombiano para la Evaluación de la Educación ICFES. (2013, diciembre). Sistema Nacional de Evaluación Estandarizada de la Educación. Alineación del examen SABER $11^{\circ}$. Bogotá, D.C.

Instituto Colombiano para la Evaluación de la Educación ICFES. (2013, diciembre). Informe de rendición de cuentas 2013. Bogotá.

Ishikawa, K. (1986). ¿Qué es el control total de calidad?: La modalidad japonesa (pp. 15 y 282). Bogotá: Editorial Norma.

ISOtools. (2012, septiembre). Colegios Acreditados en NEASC-CIS. República Dominicana. Recuperado de http://www.isotools.org/2012/09/28/colegiosacreditados-en-neasc-cis-garantia-de-calidad-educativa/\#sthash.4k3txfL1.dpuf

Juran, J. (1994). Juran Joseph y la calidad por el diseño. Editorial Díaz de Santos.

Juran. J., Godfrey, M., y Blanton, A. (2001). Manual de calidad. (5ta ed., vol. I., pp. 3 y 7). España: Editorial Mc Graw-Hill.

León, R. G. (2008). Evaluación del programa escuelas de calidad en el estado de Puebla: Resultados sobre logro educativo. Revista Mexicana De Investigación Educativa, 13(38), 891-917. Recuperado de http://search.proquest. com/docview/199317704?accountid=36216

López, N. E. (2007). Acerca de la problemática de la evaluación de la calidad de la educación en Colombia. Universidad de Caldas. ProQuest ebrary.

Martínez de D., E. y otra. (2002). La investigación sobre la educación superior en Colombia. Un estado del arte (p. 28). ICFES. Bogotá.

Martínez, C., y Riopérez, N. (2005). El modelo de excelencia en la EFQM y su aplicación para la mejora de la calidad de los centros educativos. (The excellence model of european foundation for quality management and its application for improving school quality). Educación XXI(8), 35-65. 
Recuperado de http://search.proquest.com/docview/1 111637808 ? accountid=36216.

Ministerio de Educación - MEN. http://www.mineducacion. gov.co/1621/w3-article-179263.html

Ministerio de Educación Nacional (2008). Guía para el mejoramiento institucional de la autoevaluación al plan de mejoramiento. Serie Guías No. 34. Revolución Educativa Colombia Aprende. Recuperado de http://www.mineducacion.gov.co/1621/articles-177745_archivo_pdf. pdf.

Ministerio de Educación Nacional. (2006). Resolución No. 4434 de 8 de agosto de 2006. "Por la cual se establecen las condiciones y mecanismos para la validación de los modelos de gestión de la calidad de los establecimientos de educación preescolar, básica y media". Recuperado de http://www.mineducacion.gov.co/1621/ articles-105282_archivo_pdf.pdf

Montes, C. (2012). Antecedentes de la normalización y acreditación en Colombia. Bogotá.

Pedraja-Rejas, L., Rodríguez-Ponce, E., Barreda, M., Sagredo, O., y Segovia, C. (2007). Estilos de liderazgo y resultados del sistema de medición de la calidad de la educación: Un estudio empírico en los colegios básicos de la ciudad de Arica, Chile. (Leadership styles and results of education quality measuring system: An empirical study in arica-chile elementary schools). Ingeniare: Revista Chilena de Ingeniería, 17(1), 21-26. Recuperado de https://www.redalyc.org/pdf/772/77211342003.pdf

Perdomo, Á. (2010). Administración de los costos y gestión financiera de la calidad (pp. 139-142). ICONTEC Internacional.

Pérez, J. A. (2010). Gestión por procesos. (4ª ed., pp. 229- 232). Madrid, España: Editorial Esic.

Rivera, J. E. (2010). Herramientas de Gestión Educativa. (1 a ed., p. 355). Bogotá: Editorial Magisterio.

Rivera, J. E. (2012). Implementación de un SGC. Información y servicios educativos para América Latina.

Rojas, J. M. (2011). Gestión Educativa en la sociedad del conocimiento. ( $2^{\mathrm{a}}$ ed., pp. 95-100).

Schargel, F. P. (2007). Cómo transformar la educación a través de la gestión de la calidad total: Guía práctica. España: Ediciones Díaz de Santos, ProQuest ebrary.

Strauss, A., y Corbin, J. (2002). Bases de la investigación cualitativa. Técnicas y procedimientos para desarrollar la teoría fundamentada. Editorial Universidad de Antioquia ( $2^{\mathrm{a}}$ ed.).

Summers, D. C.S. (2006). Administración de la calidad. México: Pearson Educación. 